\title{
Comparison of activation patterns between masking and inattention tasks: a coordinate-based meta-analysis of implicit emotional face processing
}

\author{
Huqing Shi ${ }^{1}$, Xiang Wang ${ }^{1}$ and Shuqiao Yao ${ }^{1,2 *}$ \\ ${ }^{1}$ Medical Psychological Institute, Second Xiangya Hospital, Central South University, Changsha, China \\ ${ }^{2}$ Key Laboratory of Psychiatry and Mental Health of Hunan Province, Second Xiangya Hospital, Central South University, Changsha, China
}

Edited by:

Hauke R. Heekeren, Freie

Universität Berlin, Germany

Reviewed by:

Agnes J. Jasinska, National Institute

on Drug Abuse, USA

Carmen Morawetz, Freie Universität

Berlin, Germany

\section{*Correspondence:}

Shuqiao Yao, Medical Psychological Institute, Second Xiangya Hospital, Central South University, 139 Middle Renmin Road, Changsha, Hunan 410011, China

e-mail: shuqiaoyao@163.com
Neuroimaging studies of implicit emotional processing are important for understanding the neural mechanisms and its social and evolutionary significance. Two major experimental tasks are used to explore the mechanisms of implicit emotional processing: masking tasks and inattention tasks, both using emotional faces as stimuli. However, it is unclear whether they have identical or distinct neural substrates since few studies have compared the two tasks. The purpose of the present study was to explore the mechanisms of implicit processing of emotional faces, and compare the activation patterns between different tasks. Through a literature search, 41 studies exploring implicit processing of emotional faces were collected. A total of 830 healthy subjects and 513 foci were obtained. Separate activation likelihood estimation (ALE) meta-analyses were conducted for the entire group of studies and for different tasks for comparison purposes. The results showed that there were differences, as well as overlap, in activation patterns between masking and inattention tasks. Bilateral amygdala, middle occipital gyrus and fusiform gyrus were activated across both tasks. While masking tasks were more associated with inferior temporal gyrus, parahippocampal gyrus and amygdala, inattention tasks were more associated with right fusiform gyrus. The differences in activation patterns between masking and inattention tasks may be indicative of separate mechanisms underlying early and late stages of implicit emotional face processing.

Keywords: emotional faces, fMRI, implicit processing, masking, inattention

\section{INTRODUCTION}

Human facial expressions are powerful non-verbal emotional cues which elicit direct and automatic responses. Many studies therefore, have focused on the neural substrate of emotional face processing. Activation of the occipital regions, middle and superior temporal gyrus, limbic regions, as well as ventral and medial prefrontal regions has been reported during facial expression processing (Haxby et al., 2000; Vuilleumier and Pourtois, 2007). Implicit processing has become a popular research interest, since it occurs in the early stages of attention and cognition, may reflect more social and evolutionary significance of emotion compared to explicit processing. Neuroimaging studies have revealed the involvement of many brain areas in implicit emotional processing, including the amygdala, thalamus, insula, the fusiform gyrus, anterior cingulate and the inferior frontal gyrus (Critchley et al., 2000a). Occipital regions such as the left lingual gyrus, right fusiform gyrus, left postcentral gyrus and right insula have also been shown to be involved (Fusar-Poli et al., 2009a). However, the above studies have employed a variety of experimental tasks and designs leading to confounding variables and disparate results.

Previous research has explored the underlying mechanism of implicit emotional processing through a variety of methods (Morris et al., 1999; Killgore and Yurgelun-Todd, 2004).
Two major tasks have been widely used to access the implicit processing, masking task and inattention task. The most popular masking task is backward masking in which a target emotional stimulus is presented for a very short period $(<40 \mathrm{~ms})$ followed immediately by a neutral stimulus such as a neutral face (as a mask) (Esteves and Ohman, 1993). Some researchers refer to this type of processing as subliminal processing, where the stimuli are attended to by the brain, but are too short or weak to be consciously perceived (Dehaene et al., 2006; Pessiglione et al., 2007, 2008). The most common contrasts used to measure neural activities for masked emotional stimuli were neutral stimuli, sometimes baseline conditions (such as fixation cross) (Pine et al., 2001; Killgore and Yurgelun-Todd, 2004; Phillips et al., 2004). The strength of masked emotional stimuli could activate subcortical regions such as the amygdala (LeDoux, 2003; Phillips et al., 2004). A rapid neural pathway from the amygdala to the early visual cortex is regarded to be involved in the subliminal emotional processing (LeDoux, 1996).

On the other hand, the most representative method of inattention task is dual-task paradigm. In this design the subject's attention is distracted by a non-emotional task such as determining the gender of the face while an emotional face is presented, or determining whether the houses (non-emotional objects) are identical while emotional faces are presented at the same time 
(Vuilleumier et al., 2001; Anderson et al., 2003). In this case, the stimuli could be consciously perceived, but stay outside of attentional focus. The attentional process is involved, but works against emotional processing by allocating attentional resources to non-emotional tasks. The most common contrasts used to measure neural activities for unattended emotional stimuli were also neutral stimuli, sometimes baseline conditions (such as fixation cross) (Lobaugh et al., 2006; Harrison et al., 2009). Some studies used contrasts such as unattended vs. attended stimuli were not investigated in this study (Williams et al., 2005; Ewbank et al., 2009). The unattended emotional stimuli could activate cortical visual cortex such as primary visual cortex (V1) (Tamietto and de Gelder, 2010). According to Kouider and Dehaene (2007), conscious perception is prevented either by insufficient stimuli strength or insufficient top-down attention, which corresponding to masking tasks and inattention tasks, respectively. That is, masking tasks reduce bottom-up input for emotional stimuli, and inattention tasks reduce top-down attention for emotional stimuli. This raises the question of whether these two tasks would lead to divergence in activation patterns of implicit emotional face processing.

Also, there has been a debate on perceptual load and emotional processing. The load theory of selective attention suggests that high perceptual load tasks have suppressive effects on taskirrelevant stimuli when the attentional resources are limited (Lavie, 2005). Some studies show reduced activities when the non-emotional tasks are demanding (Pessoa et al., 2002, 2005; Pessoa, 2005; Silvert et al., 2007). However, it remains unclear whether the implicit emotional processing would interact with different levels of perceptual load.

Based on the above question, the aim of the current study was to examine effects of experimental tasks on brain activation of emotional stimuli through an activation likelihood estimation (ALE) meta-analysis of fMRI studies (Turkeltaub et al., 2002). This method has the advantage of increasing sample size and extracting specific activated brain areas from different studies to understand a full representation of the activation patterns. Masking tasks and inattention tasks both reveal underlying mechanisms of implicit emotional processing but place emphasis on different stages. The present study analyzed these two processes together and separately. Sub-analyses for different perceptual load were also carried out to examine possible effects. Based on previous research, we hypothesized that the brain activation patterns would differ between tasks. Masking tasks would be more involved with sub-cortical areas such as amygdala; inattention tasks would be more involved with cortical areas such as prefrontal cortex. Also, there would be overlapping areas showing consistent activation patterns for implicit emotional face processing.

\section{METHODS}

\section{LITERATURE SEARCH AND INCLUSION CRITERIA}

An online search of journal articles from January 1993 to June 2013 via PubMed, Web of Science was conducted by using key words "emotional face," "fMRI," "implicit," "covert," "masking," "inattention," "unattended," "distract," "dual-task," etc. Each being fully examined, articles were selected based on the following inclusion criteria: (1) fMRI studies published in peer reviewed journals with healthy subjects; (2) used emotional faces as experimental stimuli; (3) used experimental paradigms implied implicit emotional processing (such as masking task, distraction task, dual-task, etc.); (4) used image subtraction methodology, results reported in emotional vs. neutral contrasts; (5) results reported as normalized spatial coordinates, either in Montreal Neurological Institute (MNI) or Talairach space. Literature were excluded for one or more reasons below: (1) used non-fMRI techniques, such as positron emission tomography (PET) or event-related potential (ERP) studies; (2) used other types of emotional stimuli (such as emotional pictures or scenes), or used faces in a non-emotional way (such as familiar faces); (3) experimental paradigms focused on psychological processes which implied only explicit processing, such as emotional faces recognition or recollection; (4) no specific emotional vs. neutral contrasts available, such as emotional vs. baseline contrasts, correlational studies or functional connectivity studies; (5) no data available in standard spatial coordinates; (6) results from case studies or reviews. Although studies with psychiatric patients were not excluded, only the results from healthy controls were included in the meta-analyses. Both negative and positive emotional stimuli were included in the meta-analyses, but separate sub-analyses were carried out to examine possible influence of emotional valence. Similarly, separate sub-analyses were carried out for event-related and block design studies, as well as sub-analyses for studies with high and low perceptual load tasks.

\section{BASIC DESCRIPTION OF INCLUDED ARTICLES}

Two reviewers independently screened the literature using the above criteria. Reference lists of the selected literature were also checked for potential inclusion. A total of 41 studies with 830 subjects, 513 foci were included in the ALE meta-analysis. Table 1 presents all the literature in this study, details are showed for: (1) demographic characteristics; (2) experimental stimuli, paradigm, and design; (3) field strength; (4) fMRI analysis methods, contrasts, and significance threshold.

\section{ACTIVATION LIKELIHOOD ESTIMATION (ALE) META-ANALYSES}

The ALE meta-analysis was carried out in standard MNI space for all selected studies, as well as separate analyses for different experimental paradigms. Foci of contrasts of emotional faces vs. neutral faces were plotted and processed. Eleven of the studies reported coordinates in Talairach space were converted into MNI space by Lancaster's transform (Lancaster et al., 2007). The whole ALE meta-analyses were accomplished by GingerALE 2.3 software (http://brainmap.org/ale/). A subject-based full-width half-maximum (FWHM) (Eickhoff et al., 2009) were applied to the data. The ALE maps were formed by statistical significance corrected for multiple comparisons at the false discovery rate (FDR) $p$-value of 0.01 and cluster extent threshold of $100 \mathrm{~mm}^{3}$ according to previous study (Sörös et al., 2009). Subanalyses for perceptual load, emotional valence, fMRI experimental design were carried out at the same statistical significance threshold (FDR $<0.01, k>100$ ), as well as sub-analyses for 


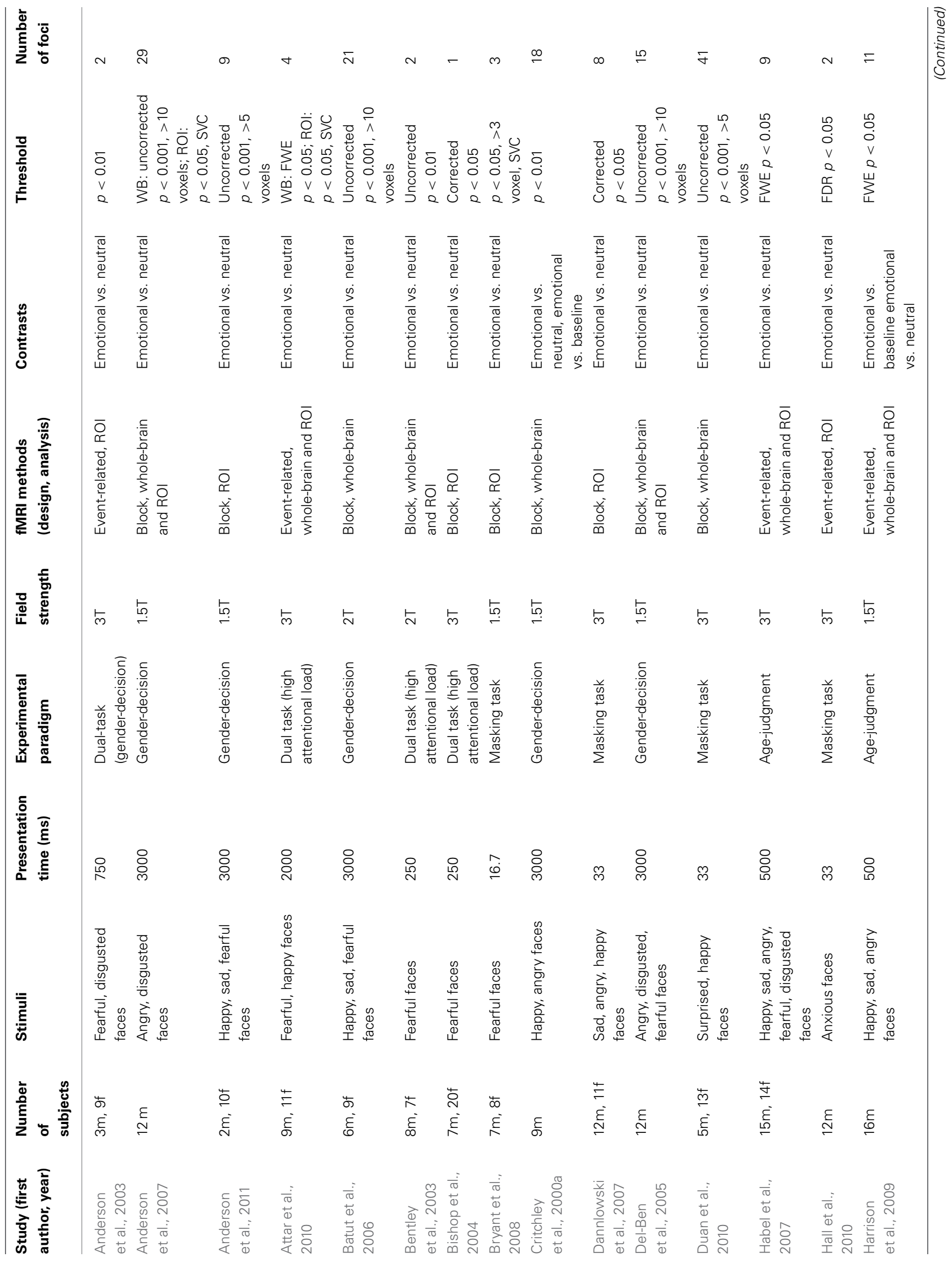




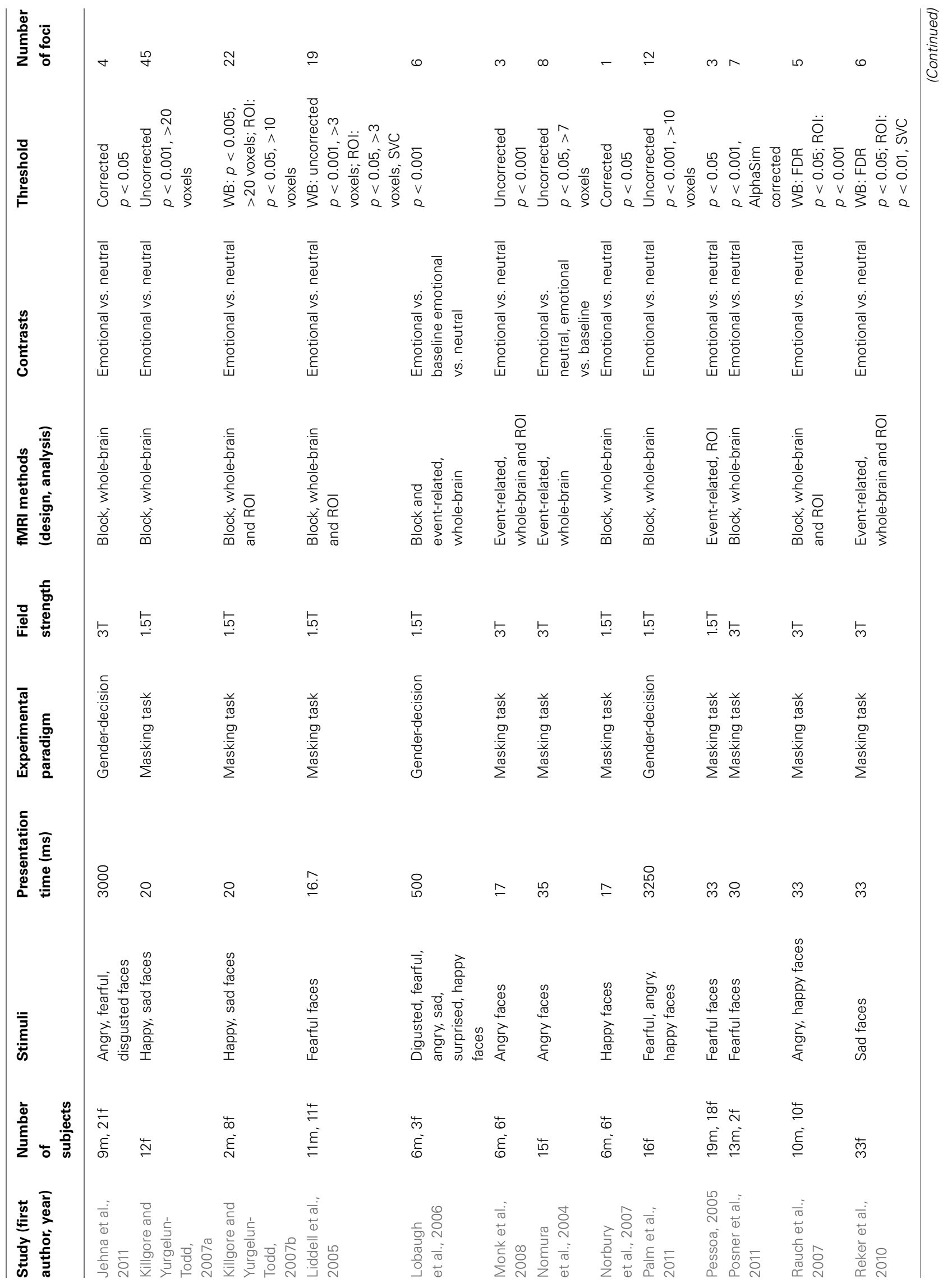




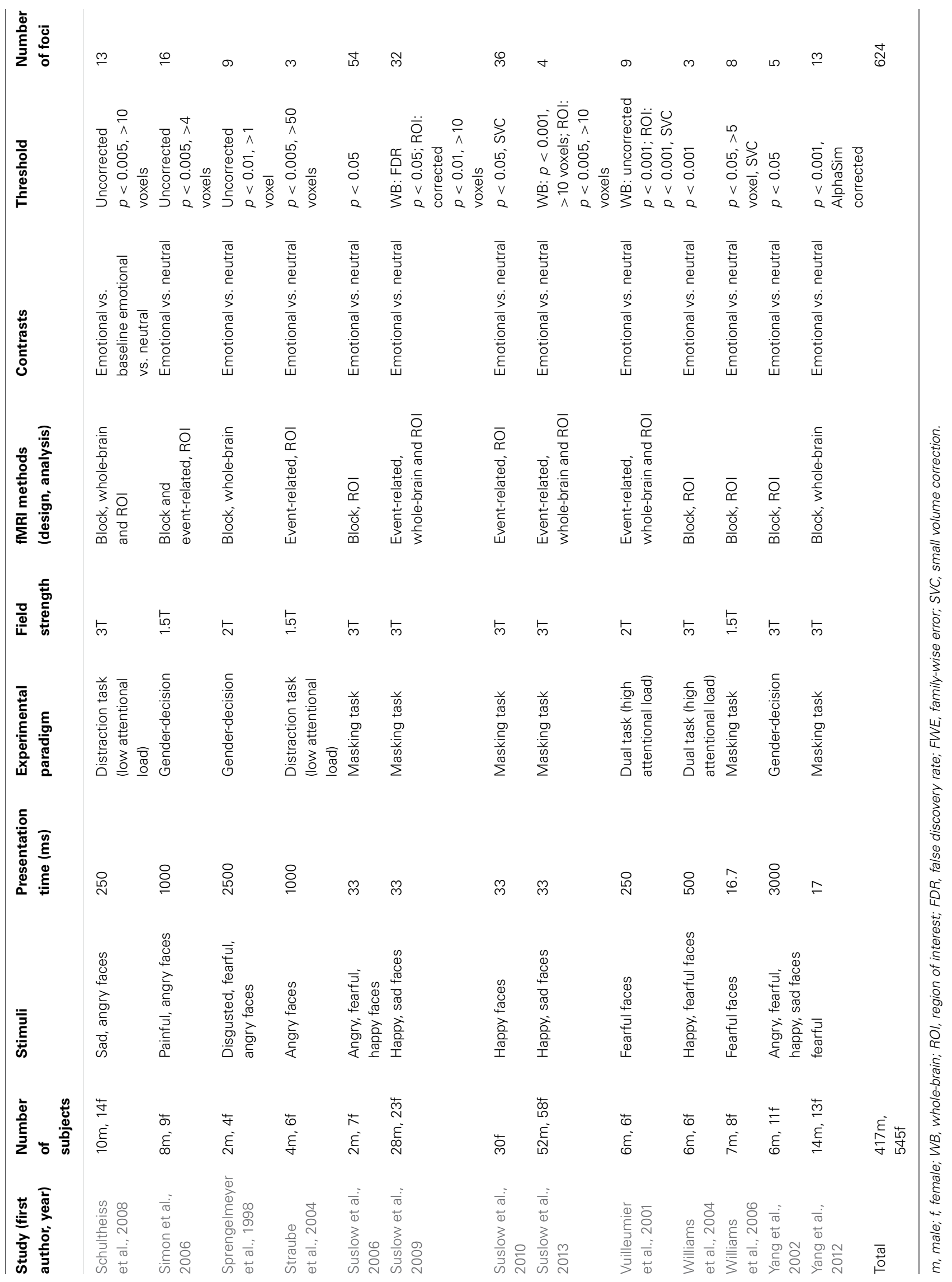


studies without using ROI analysis. The comparison between the ALE maps generated by different tasks was obtained by subtraction of ALE values in each voxel using GingerALE too (Eickhoff et al., 2011). A permutation testing with 5000 iterations was made and comparison ALE maps were formed at FDR $p$-value of 0.05 , minimum cluster size of $100 \mathrm{~mm}^{3}$. All maps of the ALE values were imported into the Mango software (http:// ric.uthscsa.edu/mango/index.html) and overlaid onto the "colinbrain" anatomical template normalized to MNI space (Kochunov et al., 2002).

\section{RESULTS}

Forty-one studies with 830 subjects and 513 foci were identified for inclusion in the ALE meta-analysis (Table 1). Four clusters were identified in the ALE analysis for all 41 studies with 78 emotional vs. neutral contrasts $(p<$ FDR $0.01, k>$ 100). As suggested in Figure 1, bilateral amygdala, right middle occipital gyrus (BA 19), and right fusiform gyrus (BA 37) were activated. In 21 studies, 55 foci indicated right amygdala activation; in 20 studies 52 foci indicated left amygdala activation, while 8 foci in one study indicated right middle occipital gyrus activation, 5 foci in 5 studies indicated right fusiform gyrus activation.

\section{MASKING TASK}

The ALE map for masking paradigms (showing 20 studies with 498 subjects and 312 foci) indicated activation in three clusters including bilateral amygdala and right middle occipital gyrus (BA 19) ( $p<$ FDR 0.01, $k>100$ ). See Figure 1. In 10 studies, 37 foci indicated right amygdala activation; 38 foci in 11 studies indicated right amygdala activation, 8 foci in one study indicated right middle occipital gyrus activation.

\section{INATTENTION TASK}

The ALE map for inattention paradigms (showing 21 studies with 332 subjects and 201 foci) indicated activation in 5 clusters, including bilateral amygdala, right fusiform gyrus (BA 37), right medial frontal gyrus (BA 10) and left insula (BA 13) ( $p$ $<$ FDR 0.01, $k>100$ ). See Figure 1. 13 foci in 10 studies indicated left amygdala activation; 5 foci in 4 studies indicated right amygdala activation; 7 foci in 7 studies indicated right fusiform gyrus activation; 3 foci in one study indicated right medial frontal gyrus activation; 2 foci in one study indicated left insula activation. All cluster details and ALE values were displayed in Table 2.

Low perceptual load tasks (showing 16 studies with 182 foci) revealed significant activation in bilateral amygdala, right fusiform gyrus (BA 37), right medial frontal gyrus (BA 10) and left insula (BA 13) ( $p<$ FDR 0.01, $k>100)$. High perceptual load tasks (showing 5 studies with 19 foci) revealed significant activation in right fusiform gyrus (BA 37), right medial frontal gyrus (BA 9), and left parahippocampal gyrus (BA 34) ( $p<$ FDR 0.01, $k>100)$.

\section{SUB-ANALYSES FOR STUDIES USING WHOLE-BRAIN ANALYSIS}

For those studies without using ROI analysis, masking tasks (showing 8 studies with 131 foci) revealed significant activation in

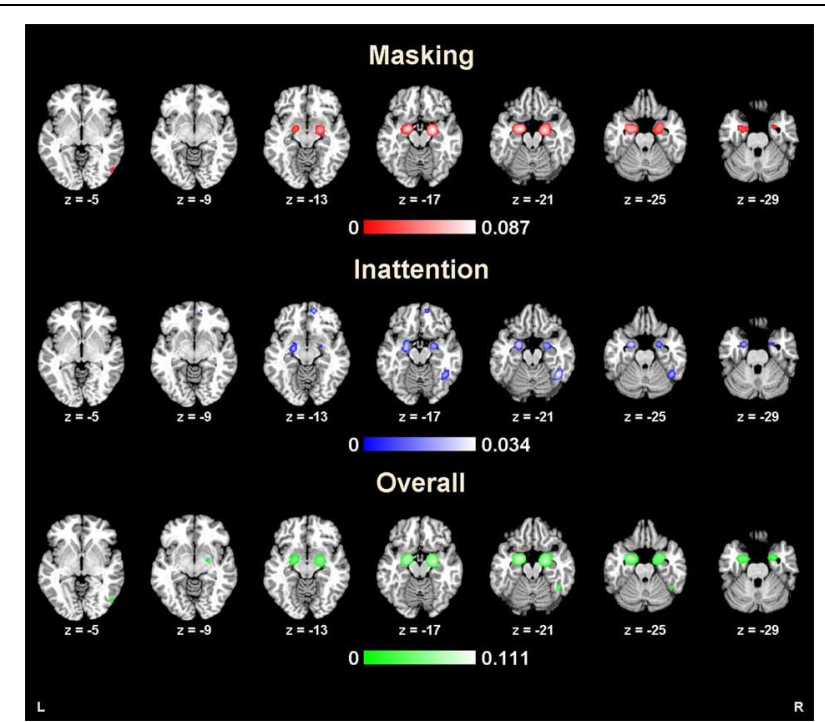

FIGURE 1 | Results from ALE analysis for masking tasks, inattention tasks, and overall studies ( $p<0.01$; FDR corrected; $k>100$ ).

bilateral middle occipital gyrus (BA 19) and right lingual gyrus ( $p$ $<$ FDR $0.01, k>100$ ). Inattention tasks (showing 12 studies with 135 foci) revealed significant activation in right fusiform gyrus (BA 37), left middle occipital gyrus (BA 18), right thalamus, left inferior frontal gyrus (BA 47) and right precuneus gyrus (BA 19) $(p<\operatorname{FDR} 0.01, k>100)$.

\section{SUB-ANALYSES FOR POSITIVE AND NEGATIVE EMOTION}

For negative emotional faces, masking tasks (showing 18 studies with 208 foci) revealed significant activation in bilateral amygdala $(p<$ FDR 0.01, $k>100$ ). Inattention tasks (showing 16 studies with 138 foci) revealed significant activation in bilateral amygdala, right fusiform gyrus, right medial frontal gyrus and left insula $(p<$ FDR 0.01, $k>100)$.

For positive emotional faces, masking tasks (showing 9 studies with 88 foci) revealed significant activation in left amygdala $(p<$ FDR $0.01, k>100$ ). Inattention tasks (showing 5 studies with 15 foci) revealed no significant activation.

\section{SUB-ANALYSES FOR EVENT-RELATED AND BLOCK DESIGN}

For event-related studies, masking tasks (showing 8 studies with 87 foci) revealed significant activation in bilateral amygdala, bilateral thalamus, left fusiform gyrus, left inferior frontal gyrus (BA 47), right postcentral gyrus (BA 3), right precuneus gyrus (BA 7), and left middle temporal gyrus (BA 21) $(p<$ FDR $0.01, k>100$ ). Inattention tasks (showing 6 studies with 38 foci) revealed significant activation in right fusiform gyrus (BA 37), right thalamus, left parahippocampal gyrus (BA 34) and right medial frontal gyrus (BA 9) $(p<\operatorname{FDR~0.01,k>}$ 100).

For block studies, masking tasks (showing 12 studies with 225 foci) revealed significant activation in bilateral amygdala and right middle occipital gyrus $(p<\operatorname{FDR} 0.01, k>100)$. Inattention 
Table 2 | ALE values of implicit processing of emotional faces.

\begin{tabular}{|c|c|c|c|c|c|c|c|}
\hline & Side & BA & $x$ & $Y$ & $z$ & Volume & ALE value \\
\hline \multicolumn{8}{|l|}{ OVERALL RESULTS } \\
\hline Amygdala & $\mathrm{R}$ & & 22 & -6 & -18 & 5128 & 0.105879 \\
\hline Middle Occipital Gyrus & $\mathrm{R}$ & 19 & 52 & -74 & -2 & 264 & 0.044556 \\
\hline Fusiform Gyrus & $\mathrm{R}$ & & 44 & -52 & -22 & 248 & 0.039371 \\
\hline Amygdala & $\mathrm{L}$ & & -18 & -4 & -20 & 3424 & 0.08703 \\
\hline Middle Occipital Gyrus & $\mathrm{R}$ & 19 & 52 & -74 & -2 & 264 & 0.044514 \\
\hline \multicolumn{8}{|l|}{ INATTENTION } \\
\hline Amygdala & $\mathrm{L}$ & & -24 & -2 & -24 & 2184 & 0.030336 \\
\hline Fusiform Gyrus & $\mathrm{R}$ & & 44 & -52 & -22 & 1464 & 0.034016 \\
\hline
\end{tabular}

Results are showed in MNI coordinates. Significance threshold is $p<F D R 0.01, k>100 . R$, right; $L$, left.

tasks (showing 15 studies with 163 foci) revealed significant activation in bilateral amygdala, right fusiform gyrus (BA 37), right medial frontal gyrus (BA 10), and left insula (BA 13) $(p<$ FDR $0.01, k>100)$.

\section{COMPARISON BETWEEN TASKS}

The comparison between two tasks obtained by subtraction revealed significantly higher right fusiform gyrus (BA37) activation for inattention tasks than masking tasks $(p<$ FDR 0.05, $k>100)$. Meanwhile, left parahippocampal gyrus, right inferior temporal gyrus and bilateral amygdala were found to be more active in masking tasks than in inattention tasks $(p<$ FDR 0.05, $k>100)$. See Figure 2 for maximal activated areas. All cluster details and $Z$-values of the subtracted image were displayed in Table 3.

\section{DISCUSSION}

The present study used ALE meta-analysis to explore brain activation in response to emotional faces by two primary implicit emotional processing tasks and paradigms. Through this method it was possible to combine studies, perform statistical analyses of the whole brain, and generate activation maps based on coordinates. To our knowledge, this is the first study to compare the mechanisms underlying the two tasks of implicit emotional processing. The two tasks of implicit processing induced activation of distinct areas, with masking tasks preferentially associated with inferior temporal gyrus and limbic areas such as parahippocampal gyrus and amygdala, while inattention tasks preferentially associated with right fusiform gyrus. Implicit emotional faces activated brain regions such as bilateral amygdala, right middle occipital gyrus and right fusiform gyrus across both tasks.

\section{IMPLICIT PROCESSING OF EMOTIONAL FACES}

Many studies have confirmed that the amygdala plays an important role in the implicit emotional face processing (Morris et al.,

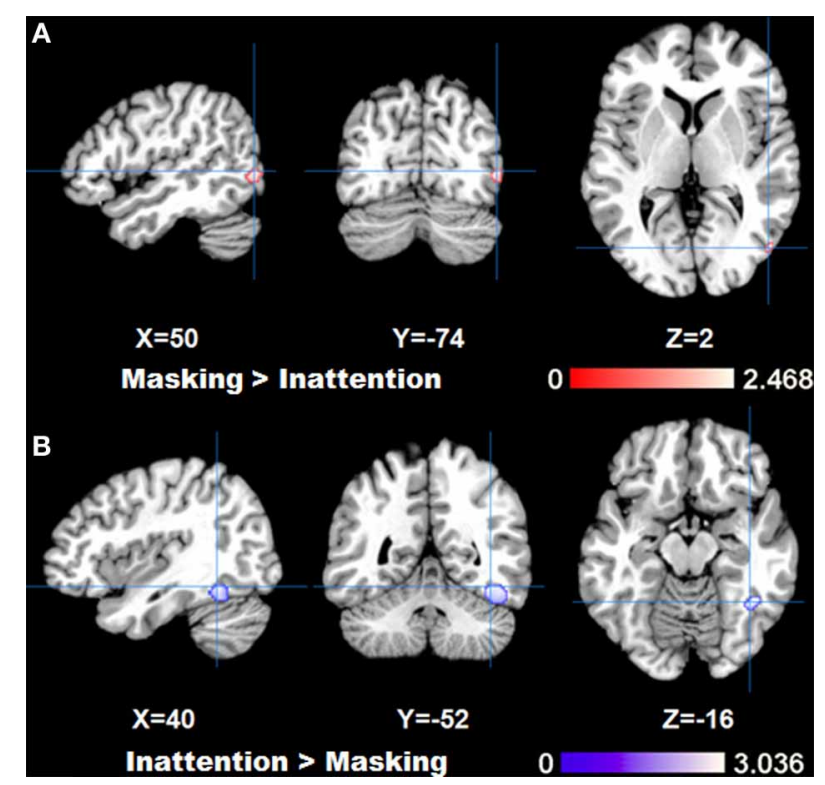

FIGURE 2 | Results of ALE analysis for comparison between tasks, (A) masking $>$ inattention task; $(B)$ inattention $>$ masking task showing areas with maximal $z$-values ( $p<0.05$; FDR corrected; $k>100$ ).

1996; Whalen et al., 1998; Williams et al., 2004). Whalen et al. (1998) regarded the amygdala as a vigilance system, functioning in conjunction with the cognition of ambiguous stimuli with biological relevance, such as emotional faces. Our overall results showed greatest activation in bilateral amygdala, which is consistent with previous studies (Baas et al., 2004; Brooks et al., 2012). According to LeDoux (1996), there exists a rapid neural pathway for salient emotional stimuli from amygdala to visual cortex. Fusiform gyrus is also regarded to be involved in this rapid neural pathway. A lot of studies have confirmed that fusiform 
Table 3 | Comparison between tasks.

\begin{tabular}{|c|c|c|c|c|c|c|c|}
\hline & Side & BA & $x$ & $Y$ & $z$ & Volume & $z$ \\
\hline \multicolumn{8}{|c|}{ INATTENTION > MASKING } \\
\hline Fusiform Gyrus & $\mathrm{R}$ & 37 & 40 & -52 & -16 & 1168 & 3.035672 \\
\hline \multicolumn{8}{|c|}{ MASKING > INATTENION } \\
\hline Parahippocampal Gyrus & $\mathrm{L}$ & 28 & -12 & -4 & -16 & 336 & 2.180776 \\
\hline Amygdala & $\mathrm{L}$ & & -34 & -2 & -24 & 168 & 2.006527 \\
\hline Amygdala & $\mathrm{R}$ & & 26 & 4 & -20 & 112 & 1.920459 \\
\hline
\end{tabular}

Results are showed in MNI coordinates. Significance threshold is $p<F D R 0.05, k>100$. R, right; L, left.

gyrus is associated with emotional face identification and perception (Morris et al., 1999; Critchley et al., 2000a; Kanwisher and Yovel, 2006; Said et al., 2011). Research also showed fusiform gyrus activation for highly salient stimuli even without conscious perception (Litt et al., 2011), which is supported by our results. Middle occipital gyrus is identified as an important part of visual cortex, thus it is not surprised to find it activated in our results for emotional face processing (Lindquist et al., 2012).

\section{COMPARISON BETWEEN TWO TASKS}

Adolphs (2002) has proposed a classification of three stages in emotional processing: first there is a rapid early processing for highly salient stimuli; then detailed processing occurs and an emotional response is aroused; third stage is when emotional recognition finally takes place. Evidence from ERP studies has revealed the time courses of these processes. Dolan (2002) found preconscious processing of emotional stimuli occurs at 100 $120 \mathrm{~ms}$ after stimulus presentation, Liddell et al. (2004) found the N2 ERP component at 200-300 ms post-stimulus range, represents an automatic, attentional response. According to Tamietto and de Gelder (2010), masking task occurs during the early stage of implicit emotional processing, while inattention task occurs during later stage of processing, especially when attention is limited (Wolbers et al., 2006). In our results, masking tasks showed only amygdala and visual area activation, while inattention tasks showed a complex network, with great activation in the fusiform gyrus, medial frontal gyrus and insula. A review by Vuilleumier and Pourtois (2007) suggested that distributed brain areas like amygdala, insula, ventral prefrontal cortex and superior temporal cortex might be involved in distinct latencies of emotional processing. Medial frontal gyrus is indicated to participate in the conscious experience of emotion (Fusar-Poli et al., 2009b). Insula is suggested to play a role in identifying the emotional significance of stimuli (Phillips et al., 2003). Paralimbic regions such as insula play an important role in conveying information between subcortical structures such as amygdala and other cortical structures (Lindquist et al., 2012). These evidence may support that inattention tasks reflect both early and later stages of emotional processing (Phelps et al., 2001). Researchers found that a subcortical pathway to the amygdala-hippocampal area for implicit emotional processing works in parallel with a cortical route to the prefrontal cortex, which is necessary for conscious identification (LeDoux, 1996; Morris et al., 1999; Phillips et al., 2003). The subcortical pathway responds rapidly to the stimuli, while the cortical pathway evaluates and regulates the response. Our results suggested that masking task preferentially activated limbic areas such as parahippocampal gyrus and amygdala, as well as inferior temporal gyrus, which is a part of visual areas, and has strong connections to the amygdala (Pessoa, 2008). Inattention task preferentially activated the fusiform gyrus, which has been stated above to be associated with emotional identification and perception. Therefore, these evidence may support that masking task reveals the early stage of emotional processing, while inattention task reveal later stage of pre-attentive processing which may serve as a transitional stage from implicit processing toward explicit processing (Phan et al., 2004).

As a priori regions of interest might be a confounding factor, we also ran a separate analysis excluding the studies using only ROI analysis. For masking tasks, studies using wholebrain analysis showed activation in visual areas such as middle occipital gyrus and lingual gyrus, which have been suggested to be involved in early processing of faces (Adolphs, 2002; Fusar-Poli et al., 2009b). For inattention tasks, activation in fusiform gyrus, middle occipital gyrus, thalamus, inferior frontal gyrus and precuneus gyrus was found. Thalamus contributes to the generation of emotional responses (Lane, 2008). Inferior frontal gyrus is known to be involved in the implicit processing of emotional faces (Adolphs, 2002; Phillips et al., 2003, 2004; Killgore and Yurgelun-Todd, 2004), and also serves as a part of core regions of ventral frontoparietal network (Corbetta et al., 2008). Precuneus gyrus is a part of superior parietal lobule, which is one of the core regions of dorsal frontoparietal network (Corbetta et al., 2008). According to Corbetta et al. (2008), ventral network is involved in directing attention to salient stimuli, and dorsal network is involved in goal-directed attentional selection. The two networks interact with each other to reorient stimulus-driven and top-down attention. Thus, this evidence also supports that inattention task reveal later stage 
of emotional processing, where pre-attentive processing takes place.

The current study emphasized the importance of experimental tasks selection in the exploration of different domains. For example, inattention tasks may be useful for studies on emotional processing influenced by attentional processes, such as attentional bias, attention deficit, and anxiety disorders (Critchley et al., 2000b; Straube et al., 2004; Anderson et al., 2007; Palm et al., 2011). The sensitivity of the masking tasks in implicit processing may make it especially useful in the detection of vulnerabilities to mental illness such as major depression disorder and subsequent primary prevention (Etkin et al., 2004; Rauch et al., 2007).

\section{SUB-ANALYSES FOR EMOTIONAL VALENCE, PERCEPTUAL LOAD, fMRI DESIGNS AND CONTRASTS}

Sub-analyses for negative emotional faces revealed almost identical results to the main results of both masking and inattention tasks. Sub-analyses for positive emotional faces revealed amygdala activation for masking tasks. However, there were not enough foci (only 15) for inattention tasks to reveal significant results. Different emotional valence was indicated to have different activation patterns (Fusar-Poli et al., 2009b; Vytal and Hamann, 2010). However, this study focused on task difference rather than emotional valence difference. Moreover, the included studies with negative emotional faces contributed to the main results for the most part. Similarly, there were not enough foci (only 19) for high perceptual load tasks to reveal reliable results. Low perceptual load tasks contributed to the main results for the most part. Future studies should include enough studies for different emotional categories and cognitive demands to evaluate the effects of emotional valence and perceptual load. Sub-analyses for block studies revealed almost identical results to the main results too. Sub-analyses for event-related studies showed variant activations other than the main results, including the thalamus, fusiform gyrus, inferior frontal gyrus, postcentral gyrus, precuneus gyrus, and middle temporal gyrus for masking tasks; thalamus and parahippocampal gyrus for inattention tasks. These regions were all suggested to be involved in emotional face processing (Brooks et al., 2012; Fusar-Poli et al., 2009b). However, this implied that heterogeneity in fMRI designs would cause bias in the meta-analysis results.

\section{LIMITATIONS}

The present study used the ALE meta-analysis method which is more reliable than a single study. The expanded sample size

\section{REFERENCES}

Adolphs, R. (2002). Recognizing emotion from facial expressions: psychological and neurological mechanisms. Behav. Cogn. Neurosci. Rev. 1, 21-62. doi: 10.1177/1534582302001001003

*Anderson, A. K., Christoff, K., Panitz, D., De Rosa, E., and Gabrieli, J. D. E. (2003). Neural correlates of the automatic processing of threat facial signals. J. Neurosci. 23, 5627-5633.
*Anderson, I. M., Del-Ben, C. M., McKie, S., Richardson, P., Williams, S. R., Elliott, R., et al. (2007). Citalopram modulation of neuronal responses to aversive face emotions: a functional MRI study. Neuroreport 18, 1351-1355. doi: 10.1097/WNR.0b013e3282742115

*Anderson, I. M., Juhasz, G., Thomas, E., Downey, D., McKie, S., Deakin, J. F., et al. (2011). The effect of acute citalopram on face emotion processing in remitted

increased statistical power. In addition, the entire scope of activation was provided. However, there were some limitations to this method of study. First, meta-analysis inherently lacks data homogeneity, thus making conclusions open to further study. Second, The ALE meta-analysis method does not account for strength of activation. It is therefore possible that important brain regions with lower activation levels may be overlooked. Third, the conversion between different coordinate systems and heterogeneous definitions of anatomical labels may also affect results.

Although masking tasks are widely accepted and used to explore implicit processing, there is no good evidence to suggest that masked faces are processed completely subliminally. Pessoa (2005) reported that more than $60 \%$ of the subjects in their study reported actually seeing the masked stimuli, indicating individual differences in sensitivity to emotional faces. Although in the current study, most of the articles using a masking task that were included in the meta-analysis provided a probe test, demonstrating that subjects were not aware of the masking stimulus. As to the inattention tasks, Phan et al. (2004) believed that tasks involving cognitive effort do not always distract attention from emotional stimuli. In fact, the fluctuation of attention could hinder the strength of comparisons made between distractors (emotional stimuli) and targets (non-emotional stimuli).

\section{CONCLUSION}

Neuroimaging studies of the implicit emotional face processing were analyzed and compared using the ALE meta-analysis method. There were distinct and overlapping results between masking tasks and inattention tasks. Masking tasks implied early stages of implicit emotional processing while inattention tasks suggested later stages of implicit emotional processing. This meta-analysis provides a new point of view to evaluate the effects of different tasks and emphasizes the importance of experimental task selection in the exploration of different domains.

\section{ACKNOWLEDGMENTS}

The present study was supported by grants from the National Key Technology R\&D Program in the 11th 5-year Plan of China (2009BA277B02 to Shuqiao Yao) and the Natural Science Foundation of China (81071104 to Shuqiao Yao, 30670709 to Shuqiao Yao).

depression: a pharmacoMRI study. Eur. Neuropsychopharmacol. 21, 140-148. doi: 10.1016/j.euroneuro.2010.06.008

*Attar, C. H., Muller, M. M., Andersen, S. K., Buchel, C., and Rose, M. (2010). Emotional processing in a salient motion context: integration of motion and emotion in both V5/hMT+ and the amygdala. J. Neurosci. 30, 5204-5210. doi: $\quad 10.1523 /$ JNEUROSCI. 5029-09.2010
Baas, D., Aleman, A., and Kahn, R. (2004). Lateralization of amygdala activation: a systematic review of functional neuroimaging studies. Brain Res. Brain Res. Rev. 45, 96-103. doi: 10.1016/j.brainresrev.2004.02.004

*Batut, A. C., Gounot, D., Namer, I. J., Hirsch, E., Kehrli, P., and Metz-Lutz, M. N. (2006). Neural responses associated with positive and negative emotion processing in patients with left versus right temporal lobe 
epilepsy. Epilepsy Behav. 9, 415-423. doi: 10.1016/j.yebeh.2006.07.013

*Bentley, P., Vuilleumier, P., Thiel, C. M., Driver, J., and Dolan, R. J. (2003). Cholinergic enhancement modulates neural correlates of selective attention and emotional processing. Neuroimage 20, 58-70. doi: 10.1016/S1053-8119(03)00302-1

*Bishop, S. J., Duncan, J., and Lawrence, A. D. (2004). State anxiety modulation of the amygdala response to unattended threat-related stimuli. J. Neurosci. 24, 10364-10368. doi: 10.1523/JNEUROSCI.2550-04.2004

Brooks, S. J., Savov, V., Allzen, E., Benedict, C., Fredriksson, R., and Schioth, H. B. (2012). Exposure to subliminal arousing stimuli induces robust activation in the amygdala, hippocampus, anterior cingulate, insular cortex and primary visual cortex: a systematic meta-analysis of fMRI studies. Neuroimage 59, 2962-2973. doi: 10.1016/j.neuroimage.2011.09.077

*Bryant, R. A., Kemp, A. H., Felmingham, K. L., Liddell, B., Olivieri, G., Peduto, A., et al. (2008). Enhanced amygdala and medial prefrontal activation during nonconscious processing of fear in posttraumatic stress disorder: an fMRI study. Hum. Brain Mapp. 29, 517-523. doi: 10.1002/hbm.20415

Corbetta, M., Patel, G., and Shulman, G. L. (2008). The reorienting system of the human brain: from environment to theory of mind. Neuron 58, 306-324. doi: 10.1016/j.neuron.2008.04.017

*Critchley, H., Daly, E., Phillips, M., Brammer, M., Bullmore, E., Williams, S., et al. (2000a). Explicit and implicit neural mechanisms for processing of social information from facial expressions: a functional magnetic resonance imaging study. Hum. Brain Mapp. 9, 93-105.

Critchley, H. D., Daly, E. M., Bullmore, E. T., Williams, S. C., Van Amelsvoort, T., Robertson, D. M., et al. (2000b). The functional neuroanatomy of social behaviour: changes in cerebral blood flow when people with autistic disorder process facial expressions. Brain 123(Pt 11), 2203-2212. doi: 10.1093/brain/123.11.2203

*Dannlowski, U., Ohrmann, P., Bauer, J., Kugel, H., Arolt, V., Heindel, W., et al. (2007). Amygdala reactivity to masked negative faces is associated with automatic judgmental bias in major depression: a $3 \mathrm{~T}$ fMR 1 study. J. Psychiatry Neurosci. 32, 423-429.

Dehaene, S., Changeux, J. P., Naccache, L., Sackur, J., and Sergent, C. (2006).
Conscious, preconscious, and subliminal processing: a testable taxonomy. Trends Cogn. Sci. 10, 204-211. doi: 10.1016/j.tics.2006.03.007

*Del-Ben, C. M., Deakin, J. F., McKie, S., Delvai, N. A., Williams, S. R., Elliott, R., et al. (2005). The effect of citalopram pretreatment on neuronal responses to neuropsychological tasks in normal volunteers: an FMRI study. Neuropsychopharmacology 30, 1724-1734. doi: 10.1038/s.npp. 1300728

Dolan, R. J. (2002). Emotion, cognition, and behavior. Science 298, 1191-1194. doi: 10.1126/science. 1076358

*Duan, X. J., Dai, Q. A., Gong, Q. Y., and Chen, H. F. (2010). Neural mechanism of unconscious perception of surprised facial expression. Neuroimage 52, 401-407. doi: 10.1016/j.neuroimage.2010.04.021

Eickhoff, S., Laird, A., Grefkes, C., Wang, L., Zilles, K., and Fox, P. (2009). Coordinate-based activation likelihood estimation meta-analysis of neuroimaging data: a random-effects approach based on empirical estimates of spatial uncertainty. Hum. Brain Mapp. 30, 2907-2926. doi: 10.1002/hbm. 20718

Eickhoff, S. B., Bzdok, D., Laird, A. R., Roski, C., Caspers, S., Zilles, K., et al. (2011). Coactivation patterns distinguish cortical modules, their connectivity and functional differentiation. Neuroimage 57, 938-949. doi: 10.1016/j.neuroimage.2011.05.021

Esteves, F., and Ohman, A. (1993). Masking the face: recognition of emotional facial expressions as a function of the parameters of backward masking. Scand. J. Psychol. 34, 1-18. doi: 10.1111/j.14679450.1993.tb01096.x

Etkin, A., Klemenhagen, K. C., Dudman, J. T., Rogan, M. T., Hen, R., Kandel, E. R., et al. (2004). Individual differences in trait anxiety predict the response of the basolateral amygdala to unconsciously processed fearful faces. Neuron 44, 1043-1055. doi: 10.1016/j.neuron.2004.12.006

Ewbank, M. P., Lawrence, A. D., Passamonti, L., Keane, J., Peers, P. V., and Calder, A. J. (2009). Anxiety predicts a differential neural response to attended and unattended facial signals of anger and fear. Neuroimage 44, 1144-1151. doi: 10.1016/j.neuroimage.2008.09.056

Fusar-Poli, P., Placentino, A., Carletti, F., Landi, P., Allen, P., Surguladze,
S., et al. (2009a). Functional atlas of emotional faces processing: a voxelbased meta-analysis of 105 functional magnetic resonance imaging studies. J. Psychiatry Neurosci. 34, 418-432.

Fusar-Poli, P., Placentino, A., Carletti, F., Landi, P., Allen, P., Surguladze, S., et al. (2009b). Functional atlas of emotional faces processing: a voxelbased meta-analysis of 105 functional magnetic resonance imaging studies. J. Psychiatry Neurosci. 34, 418-432.

*Habel, U., Windischberger, C., Derntl, B., Robinson, S., Kryspin-Exner, I., Gur, R. C., et al. (2007). Amygdala activation and facial expressions: explicit emotion discrimination versus implicit emotion processing. Neuropsychologia 45, 2369-2377. doi: $\quad 10.1016 / j . n e u r o p s y c h o l o g i a$. 2007.01.023

*Hall, G. B., Doyle, K. A., Goldberg, J., West, D., and Szatmari, P. (2010). Amygdala engagement in response to subthreshold presentations of anxious face stimuli in adults with autism spectrum disorders: preliminary insights. PLoS ONE 5:e10804. doi: 10.1371/journal.pone.0010804

*Harrison, N. A., Brydon, L., Walker C., Gray, M. A., Steptoe, A., and Critchley, H. D. (2009). Inflammation causes mood changes through alterations in subgenual cingulate activity and mesolimbic connectivity. Biol. Psychiatry 66, 407-414. doi: 10.1016/j.biopsych.2009.03.015

Haxby, J. V., Hoffman, E. A., and Gobbini, M. I. (2000). The distributed human neural system for face perception. Trends Cogn. Sci. 4 , 223-233. doi: 10.1016/S1364-6613 (00)01482-0

*Jehna, M., Neuper, C., Ischebeck, A., Loitfelder, M., Ropele, S., Langkammer, C., et al. (2011). The functional correlates of face perception and recognition of emotional facial expressions as evidenced by fMRI. Brain Res. 1393, 73-83. doi: 10.1016/j.brainres.2011.04.007

Kanwisher, N., and Yovel, G. (2006). The fusiform face area: a cortical region specialized for the perception of faces. Philos. Trans. R. Soc. Lond. B Biol. Sci. 361, 2109-2128. doi: 10.1098/rstb.2006.1934

Killgore, W. D., and Yurgelun-Todd, D. A. (2004). Activation of the amygdala and anterior cingulate during nonconscious processing of sad versus happy faces. Neuroimage 21, 1215-1223. doi: 10.1016/j.neuroimage.2003.12.033

*Killgore, W. D., and YurgelunTodd, D. A. (2007a). Unconscious processing of facial affect in children and adolescents. Soc. Neurosci. 2, 28-47. doi: 10.1080/ 17470910701214186

*Killgore, W. D. S., and YurgelunTodd, D. A. (2007b). The right-hemisphere and valence hypotheses: could they both be right (and sometimes left)? Soc. Cogn. Affect. Neurosci. 2, 240-250. doi: $10.1093 /$ scan/nsm020

Kochunov, P., Lancaster, J., Thompson, P., and Toga, A. (2002). An optimized individual target brain in the Talairach coordinate system. Neuroimage. 17, 922-927. doi: 10.1006/nimg.2002.1084

Kouider, S., and Dehaene, S. (2007). Levels of processing during non-conscious perception: a critical review of visual masking. Philos. Trans. R. Soc. Lond. B Biol. Sci. 362, 857-875. doi: 10.1098/rstb.2007.2093

Lancaster, J., Tordesillas-Gutiérrez, D., Martinez, M., Salinas, F., Evans, A., Zilles, K., et al. (2007). Bias between MNI and Talairach coordinates analyzed using the ICBM-152 brain template. Hum. Brain Mapp. 28, 1194-1205. doi: 10.1002/hbm.20345

Lane, R. D. (2008). Neural substrates of implicit and explicit emotional processes: a unifying framework for psychosomatic medicine. Psychosom. Med. 70, 214-231. doi: 10.1097/PSY.0b013e3181647e44

Lavie, N. (2005). Distracted and confused?: selective attention under load. Trends Cogn. Sci. 9, 75-82. doi: 10.1016/j.tics.2004.12.004

LeDoux, J. (1996). Emotional networks and motor control: a fearful view. Prog. Brain Res. 107, 437-446. doi: 10.1016/S0079-6123(08)61880-4

LeDoux, J. (2003). The emotional brain, fear, and the amygdala. Cell. Mol. Neurobiol. 23, 727-738. doi: 10.1023/A:1025048802629

*Liddell, B. J., Brown, K. J., Kemp, A. H., Barton, M. J., Das, P., Peduto, A., et al. (2005). A direct brainstemamygdala-cortical 'alarm' system for subliminal signals of fear. Neuroimage 24, 235-243. doi: 10.1016/j.neuroimage.2004.08.016

Liddell, B. J., Williams, L. M., Rathjen, J., Shevrin, H., and Gordon, E. (2004). A temporal dissociation of subliminal versus supraliminal fear perception: an event-related potential study. J. Cogn. Neurosci. 16, 479-486. doi $10.1162 / 089892904322926809$

Lindquist, K. A., Wager, T. D., Kober, H., Bliss-Moreau, E., and Barrett, L. F. (2012). The brain basis of emotion: a meta-analytic review. 
Behav. Brain Sci. 35, 121-143. doi: 10.1017/S0140525X11000446

Litt, A., Plassmann, H., Shiv, B., and Rangel, A. (2011). Dissociating valuation and saliency signals during decision-making. Cereb. Cortex 21, 95-102. doi: 10.1093/cercor/bhq065

*Lobaugh, N. J., Gibson, E., and Taylor, M. J. (2006). Children recruit distinct neural systems for implicit emotional face processing. Neuroreport 17, 215-219. doi: 10.1097/01.wnr.0000198946. 00445.2f

*Monk, C. S., Telzer, E. H., Mogg, K., Bradley, B. P., Mai, X. Q., Louro, H. M. C., et al. (2008). Amygdala and ventrolateral prefrontal cortex activation to masked angry faces in children and adolescents with generalized anxiety disorder. Arch. Gen. Psychiatry 65, 568-576. doi: 10.1001/archpsyc.65.5.568

Morris, J. S., Frith, C. D., Perrett, D. I., Rowland, D., Young, A. W., Calder, A. J., et al. (1996). A differential neural response in the human amygdala to fearful and happy facial expressions. Nature 383, 812-815. doi: 10.1038/383812a0

Morris, J. S., Ohman, A., and Dolan, R. J. (1999). A subcortical pathway to the right amygdala mediating unseen fear. Proc. Natl. Acad. Sci. U.S.A. 96, 1680-1685. doi: 10.1073/pnas.96.4.1680

*Nomura, M., Ohira, H., Haneda, K., Iidaka, T., Sadato, N., Okada, T., et al. (2004). Functional association of the amygdala and ventral prefrontal cortex during cognitive evaluation of facial expressions primed by masked angry faces: an event-related fMRI study. Neuroimage 21, 352-363. doi: $\quad 10.1016 /$ j.neuroimage.2003. 09.021

*Norbury, R., Mackay, C. E., Cowen, P. J., Goodwin, G. M., and Harmer, C. J. (2007). Short-term antidepressant treatment and facial processing. functional magnetic resonance imaging study. $\mathrm{Br}$. J. Psychiatry 190, 531-532. doi: 10.1192/bjp.bp.106.031393

*Palm, M. E., Elliott, R., Mckie, S., Deakin, J. F. W., and Anderson, I. M. (2011). Attenuated responses to emotional expressions in women with generalized anxiety disorder. Psychol. Med. 41, 1009-1018. doi: 10.1017/S0033291710001455

Pessiglione, M., Petrovic, P., Daunizeau, J., Palminteri, S., Dolan, R. J., and Frith, C. D. (2008). Subliminal instrumental conditioning demonstrated in the human brain. Neuron 59, 561-567. doi: 10.1016/j.neuron.2008.07.005
Pessiglione, M., Schmidt, L., Draganski, B., Kalisch, R., Lau, H., Dolan, R. J., et al. (2007). How the brain translates money into force: a neuroimaging study of subliminal motivation. Science 316, 904-906. doi: 10.1126/science. 1140459

Pessoa, L. (2005). To what extent are emotional visual stimuli processed without attention and awareness? Curr. Opin. Neurobiol. 15, 188-196. doi: 10.1016/j.conb.2005.03.002

Pessoa, L. (2008). On the relationship between emotion and cognition. Nat. Rev. Neurosci. 9, 148-158. doi: $10.1038 / \mathrm{nrn} 2317$

Pessoa, L., Kastner, S., and Ungerleider, L. G. (2002). Attentional control of the processing of neural and emotional stimuli. Brain Res. Cogn. Brain Res. 15, 31-45. doi: 10.1016/S0926-6410(02)00214-8

* Pessoa, L., Padmala, S., and Morland, T. (2005). Fate of unattended fearful faces in the amygdala is determined by both attentional resources and cognitive modulation. Neuroimage 28, 249-255. doi: 10.1016/j.neuroimage.2005.05.048

Phan, K. L., Wager, T. D., Taylor, S. F., and Liberzon, I. (2004). Functional neuroimaging studies of human emotions. CNS Spectr. 9, 258-266.

Phelps, E., O'Connor, K., Gatenby, J., Gore, J., Grillon, C., and Davis, M. (2001). Activation of the left amygdala to a cognitive representation of fear. Nat. Neurosci. 4, 437-441. doi: 10.1038/86110

Phillips, M. L., Drevets, W. C., Rauch, S. L., and Lane, R. (2003). Neurobiology of emotion perception I: the neural basis of normal emotion perception. Biol. Psychiatry 54, 504-514. doi: 10.1016/S0006-3223(03)00168-9

Phillips, M. L., Williams, L. M., Heining, M., Herba, C. M., Russell, T., Andrew, C., et al. (2004). Differential neural responses to overt and covert presentations of facial expressions of fear and disgust. Neuroimage 21, 1484-1496. doi: 10.1016/j.neuroimage.2003.12.013

Pine, D. S., Grun, J., Zarahn, E., Fyer, A., Koda, V., Li, W., et al. (2001). Cortical brain regions engaged by masked emotional faces in adolescents and adults: an fMRI study. Emotion 1, 137-147. doi: 10.1037/1528-3542.1.2.137

*Posner, J., Nagel, B. J., Maia, T. V., Mechling, A., Oh, M., Wang, Z., et al. (2011). Abnormal amygdalar activation and connectivity in adolescents with attentiondeficit/hyperactivity disorder. J. Am.
Acad. Child Adolesc. Psychiatry 50, 828-837, e823.

*Rauch, A. V., Ohrmann, P., Bauer, J., Kugel, H., Engelien, A., Arolt, V., et al. (2007). Cognitive coping style modulates neural responses to emotional faces in healthy humans: a 3-t fMRI study. Cereb. Cortex 17, 2526-2535. doi: 10.1093/cercor/bhl158

* Reker, M., Ohrmann, P., Rauch, A. V., Kugel, H., Bauer, J., Dannlowski, U., et al. (2010). Individual differences in alexithymia and brain response to masked emotion faces. Cortex 46, 658-667. doi: 10.1016/j.cortex.2009.05.008

Said, C., Haxby, J., and Todorov, A. (2011). Brain systems for assessing the affective value of faces. Philos. Trans. R. Soc. Lond. B Biol. Sci. 366, 1660-1670. doi: 10.1098/rstb.2010.0351

*Schultheiss, O. C., Wirth, M. M., Waugh, C. E., Stanton, S. J., Meier, E. A., and Reuter-Lorenz, P. (2008). Exploring the motivational brain: effects of implicit power motivation on brain activation in response to facial expressions of emotion. Soc. Cogn. Affect. Neurosci. 3, 333-343. doi: 10.1093/scan/nsn030

Silvert, L., Lepsien, J., Fragopanagos, N., Goolsby, B., Kiss, M., Taylor, J. G., et al. (2007). Influence of attentional demands on the processing of emotional facial expressions in the amygdala. Neuroimage 38, 357-366. doi: 10.1016/j.neuroimage.2007.07.023

* Simon, D., Craig, K. D., Miltner, W. H. R., and Rainville, P. (2006). Brain responses to dynamic facial expressions of pain. Pain 126, 309-318. doi: 10.1016/j.pain. 2006.08.033

Sörös, P., Inamoto, Y., and Martin, R. (2009). Functional brain imaging of swallowing: an activation likelihood estimation meta-analysis. Hum. Brain Mapp. 30, 2426-2439. doi: 10.1002/hbm.20680

*Sprengelmeyer, R., Rausch, M., Eysel, U. T., and Przuntek, H. (1998). Neural structures associated with recognition of facial expressions of basic emotions. Proc. Biol. Sci. 265, 1927-1931. doi: 10.1098/rspb.1998.0522

*Straube, T., Kolassa, I. T., Glauer, M., Mentzel, H. J., and Miltner, W. H. R. (2004). Effect of task conditions on brain responses to threatening faces in social phobics: an event-related functional magnetic resonance Imaging study. Biol. Psychiatry 56, 921-930. doi: $\quad$ 10.1016/j.biopsych.2004. 09.024
*Suslow, T., Konrad, C., Kugel, H., Rumstadt, D., Zwitserlood, P., Schoning, S., et al. (2010). Automatic mood-congruent amygdala responses to masked facial expressions in major depression. Biol. Psychiatry 67, 155-160. doi: 10.1016/j.biopsych.2009.07.023

*Suslow, T., Kugel, H., Ohrmann, P., Stuhrmann, A., Grotegerd, D., Redlich, R., et al. (2013). Neural correlates of affective priming effects based on masked facial emotion: an fMRI study. Psychiatry Res. 211, 239-245. doi: $\quad 10.1016 /$ j.pscychresns.2012. 09.008

*Suslow, T., Kugel, H., Rauch, A. V., Dannlowski, U., Bauer, J., Konrad, C., et al. (2009). Attachment avoidance modulates neural response to masked facial emotion. Hum. Brain Mapp. 30, 3553-3562. doi: 10.1002/hbm. 20778

* Suslow, T., Ohrmann, P., Bauer, J., Rauch, A. V., Schwindt, W., Arolt, V., et al. (2006). Amygdala activation during masked presentation of emotional faces predicts conscious detection of threatrelated faces. Brain Cogn. 61, 243-248. doi: 10.1016/j.bandc. 2006.01.005

Tamietto, M., and de Gelder, B. (2010). Neural bases of the non-conscious perception of emotional signals. Nat. Rev. Neurosci. 11, 697-709. doi: 10.1038/nrn2889

Turkeltaub, P. E., Eden, G. F., Jones, K. M., and Zeffiro, T. A. (2002). Meta-analysis of the functional neuroanatomy of single-word reading: method and validation. Neuroimage 16, 765-780. doi: 10.1006/nimg.2002.1131

*Vuilleumier, P., Armony, J., Driver, J., and Dolan, R. (2001). Effects of attention and emotion on face processing in the human brain: an event-related fMRI study. Neuron 30, 829-841. doi: 10.1016/S08966273(01)00328-2

Vuilleumier, P., and Pourtois, G. (2007). Distributed and interactive brain mechanisms during emotion face perception: evidence from functional neuroimaging. Neuropsychologia 45, 174-194. doi: $10.1016 /$ j.neuropsychologia 2006.06.003

Vytal, K., and Hamann, S. (2010). Neuroimaging support for discrete neural correlates of basic emotions: a voxel-based meta-analysis. J. Cogn. Neurosci. 22, 2864-2885. doi: 10.1162/jocn.2009.21366

Whalen, P. J., Rauch, S. L., Etcoff, N. L., McInerney, S. C., Lee, M. B., and Jenike, M. A. (1998). 
Masked presentations of emotional facial expressions modulate amygdala activity without explicit knowledge. J. Neurosci. 18, 411-418.

*Williams, L. M., Das, P., Liddell, B. J., Kemp, A. H., Rennie, C. J., and Gordon, E. (2006). Mode of functional connectivity in amygdala pathways dissociates level of awareness for signals of fear. J. Neurosci. 26, 9264-9271. doi: 10.1523/ JNEUROSCI.1016-06.2006

Williams, M. A., McGlone, F., Abbott, D. F., and Mattingley, J. B. (2005). Differential amygdala responses to happy and fearful facial expressions depend on selective attention. Neuroimage 24, 417-425. doi: 10.1016/j. neuroimage.2004.08.017
*Williams, M. A., Morris, A. P., McGlone, F., Abbott, D. F., and Mattingley, J. B. (2004). Amygdala responses to fearful and happy facial expressions under conditions of binocular suppression. J. Neurosci. 24, 2898-2904. doi: 10.1523/JNEUROSCI.4977-03.2004

Wolbers, T., Schoell, E. D., Verleger, R., Kraft, S., McNamara, A., Jaskowski, P., et al. (2006). Changes in connectivity profiles as a mechanism for strategic control over interfering subliminal information. Cereb. Cortex 16, 857-864. doi: 10.1093/cercor/bhj029

*Yang, J., Cao, Z., Xu, X., and Chen, G. (2012). The amygdala is involved in affective priming effect for fearful faces. Brain Cogn. 80, 15-22. doi: 10.1016/j.bandc.2012. 04.005
*Yang, T. T., Menon, V., Eliez, S., Blasey, C., White, C. D., Reid, A. J., et al. (2002). Amygdalar activation associated with positive and negative facial expressions. Neuroreport 13, 1737-1741. doi: 10.1097/00001756-20021007000009

Conflict of Interest Statement: The authors declare that the research was conducted in the absence of any commercial or financial relationships that could be construed as a potential conflict of interest.

Received: 21 March 2013; accepted: 24 July 2013; published online: 26 August 2013
Citation: Shi H, Wang $X$ and Yao $S$ (2013) Comparison of activation patterns between masking and inattention tasks: a coordinate-based meta-analysis of implicit emotional face processing. Front. Hum. Neurosci. 7:459. doi: 10.3389/fnhum.2013.00459

This article was submitted to the journal Frontiers in Human Neuroscience.

Copyright (c) 2013 Shi, Wang and Yao. This is an open-access article distributed under the terms of the Creative Commons Attribution License (CC BY).

The use, distribution or reproduction in other forums is permitted, provided the original author(s) or licensor are credited and that the original publication in this journal is cited, in accordance with accepted academic practice. No use, distribution or reproduction is permitted which does not comply with these terms.

* References marked with an asterisk indicate studies included in the meta-analysis. 